\title{
Inmigración, diferencias culturales y accidentes de trabajo
}

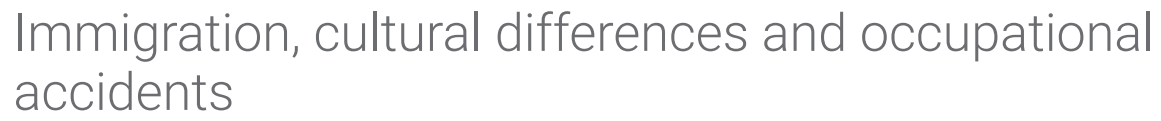

José A. García-Arroyo ${ }^{1}$

'Departamento de Psicología Social, UNED, Madrid, España.

Fechas · Dates

Recibido: 2020.11.01

Publicado: 2021.01.21
Sección coordinada por / Section Coordinator

Dr. Guillermo García González

Profesor Titular Derecho del Trabajo y SS Universidad Internacional de La Rioja (UNIR)

Email: guillermo.garcia@unir.net 


\section{Entrevista a José A. García-Arroyo}

\section{1. ¿Cuál es la procedencia, especialización y áreas de trabajo de los autores del artículo? ¿Qué características le parecen más destacables de este grupo de trabajo?}

Los autores procedemos del área de Psicología del Trabajo y de las Organizaciones de la Universidad Nacional de Educación a Distancia, y nos dedicamos a la investigación y docencia en el ámbito de la salud laboral y de la gestión de recursos humanos. En los últimos años, nos hemos centrado en el estudio de los riesgos psicosociales y su influencia sobre la salud y los accidentes. Concretamente, el artículo de referencia se centra en el papel de la cultura y de las diferencias culturales en los accidentes de trabajo.

No obstante, aunque este artículo lo firmamos dos autores, José Antonio García-Arroyo y Amparo Osca, nuestro equipo de trabajo es más amplio e incluye profesores de la Universidad Politécnica de Valencia y de la Universidad de Navarra. También colaboramos con profesionales y profesores de universidades latinoamericanas, lo que enriquece nuestro estudio con la variedad cultural. La colaboración entre investigadores y consultores aporta una visión sobre las organizaciones muy cercana a lo que ocurre en la realidad, lo que constituye una fortaleza, tanto desde el punto de vista teórico como aplicado.

\section{2. ¿Cómo se ha financiado el estudio?}

Este estudio no tiene financiación formal, aunque debemos agradecer a la Secretaría General de Estadística del Ministerio de Trabajo y Economía Social que nos proporcionaran los datos de accidentabilidad con los que hemos trabajado. Estos incluyen información de más de 40.000 accidentes de trabajo de inmigrantes de 38 países. Además, hemos cruzado estos datos con los aportados por Hosftede en su página web (www.hofstede-insights.com) sobre los valores culturales de los diferentes países de mundo. Es decir, hemos trabajado con dos bases de datos que son accesibles para cualquier investigador y tremendamente valiosas. Los "datos son el petróleo del siglo XXI porque, cuándo se trabaja con información tan rigurosa y con muestras tan grandes, van a generar resultados relevantes.

\section{3. ¿Qué problema pretende abordar este estudio y dónde radica su interés o relevancia para la seguridad y salud en el trabajo?}

Los accidentes de trabajo son, por definición, un fenómeno policausal y, por tanto, difícil de abordar en toda su complejidad. En este caso nosotros planteamos una perspectiva cultural de los accidentes. ¿Por qué esta perspectiva? El estudio de los valores culturales se inicia en los años 80 del siglo pasado con trabajos como el de G.H. Hofstede en la empresa IBM. Este autor entiende la cultura como la programación colectiva de la mente que influye en la forma de pensar, sentir y actuar y distingue a los miembros de un grupo de otros. En un primer momento, el autor propone cinco valores culturales: individualismo vs colectivismo, distancia de poder, aversión a la incertidumbre, masculinidad vs feminidad y orientación a largo plazo. Estos valores se han relacionado con aspectos tan importantes como 
los estilos de liderazgo, el emprendimiento o la innovación. En nuestro artículo relacionamos los valores culturales con los accidentes de trabajo, algo que ya se ha hecho en otros países, pero no en el nuestro, por eso creemos que es importante.

Además, la integración de la población inmigrante es un reto social de primer orden porque su contribución es fundamental para la economía de muchos países y porque sus tasas de accidentalidad son elevadas. Ya se ha señalado su mayor vulnerabilidad, sobre todo, porque sus condiciones laborales suelen ser más precarias, pero también hay otras variables que pueden estar influyendo y la cultura es una de ellas.

La relevancia de nuestro estudio radica en que compara el peso de tres variables culturales, algo que no es frecuente. Primero, analiza el papel del idioma del país origen de los emigrantes. pues, en muchas ocasiones, los accidentes se atribuyen a la dificultad para entender las instrucciones o la normativa en materia de salud laboral. En segundo lugar, incluye los valores culturales del país de origen, siguiendo la clasificación de Hofstede; y, por último, estudia también las diferencias entre los valores del país de origen y los valores de España. Este triple abordaje nos permite obtener resultados que creemos muy interesantes. Contrariamente a lo que se podía esperar, no se accidentan más los trabajadores que provienen de países con idiomas diferentes al español y, lo que es más importante, tampoco se accidentan más los trabajadores que provienen de países con valores culturales más diferentes a los españoles.

\section{4. ¿Qué aporta este estudio de novedoso o destacable en relación con el resto de producción científica sobre el problema estudiado?}

Además de lo que ya hemos señalado, podemos destacar otros dos aspectos novedosos. Por una parte, hemos distinguido el sector económico en el que trabajan los emigrantes y hemos probado diferentes tipos de relaciones: lineales y no lineales.

Respecto al sector económico, parece claro que, si no se distingue entre sectores, lo que ocurre en un sector pueden anular lo que ocurre en otro. En materia de salud laboral no es lo mismo lo que sucede en el sector agrícola y lo que sucede en la construcción.

Respecto al tipo de relaciones, se sabe que el comportamiento de las variables no es siempre lineal; es decir, el efecto de una variable no siempre crece o disminuye de manera uniforme, sino que puede haber relaciones curvilíneas e incluso interacciones entre variables. Esto que es ampliamente aceptado en la investigación en general, apenas se ha estudiado en este ámbito, por eso creemos que es importante. Nuestros resultados muestran que las relaciones entre los valores culturales del país de origen y los accidentes siguen, sobre todo, patrones curvilíneos. Destacar, por ejemplo, el papel del valor distancia de poder. Como se sabe, los empleados de países con baja distancia de poder prefieren relaciones más igualitarias con sus superiores y viceversa. Según nuestros resultados, la tasa más baja de accidentes se da en empleados de países que puntúan alrededor de 30 (según la clasificación de Hosftede). A medida que aumenta la puntuación en distancia de 
poder aumenta también la tasa de accidentes, pero solo hasta un punto, alrededor del 70, porque a partir de ahí esta relación es inversa, es decir, a más distancia de poder menos accidentes. Lo mismo ocurre en el sector servicios y, en ambos casos, esa relación explica porcentajes de varianza de los accidentes importantes.

En la industria se observa otro patrón interesante respecto a la distancia de poder. En este sector, tienen más accidentes los empleados que provienen de países con baja distancia de poder y bajo individualismo, porque cuando los trabajadores proceden de países con baja distancia de poder y alto individualismo, su tasa de accidentes tiende a ser menor.

\section{5. ¿Se han encontrado con alguna dificultad o contratiempo para el desarrollo del estudio?}

La única dificultad es que cuándo se comenzó el estudio, los últimos datos disponibles sobre accidentabilidad laboral eran los del 2015, el estudio se realizó durante el año 2017, se envió a la revista en 2018 y finalmente se publicó en octubre de 2019. Aunque nosotros fuimos relativamente rápidos en realizar el estudio, las revistas de impacto tienen un proceso de publicación que se alarga demasiado. En algunas investigaciones esta demora puede hacer que los resultados queden anticuados antes de publicarse. Afortunadamente, en nuestro caso al tratarse de un estudio con un enfoque cultural, no afecta demasiado, pues la cultura tarda mucho en cambiar; aun así, el lapso de tiempo entre la investigación y su publicación constituye uno de los problemas de la investigación actual.

\section{6. ¿Se han obtenido los resultados esperados o se ha producido alguna sorpre- sa?}

En general, como hemos planteado, constatamos sobre todo relaciones curvilíneas entre las características culturales y los accidentes de trabajo, y que estas relaciones son diferentes según el sector económico. No todos los trabajadores inmigrantes se accidentan más que los trabajadores locales, pero las características culturales de su país de origen sí se relacionan con sus tasas de accidentabilidad.

Otro resultado que no esperábamos, pero que queremos resaltar es que, en general, provenir de un país donde se habla un idioma diferente no supone accidentarse más.

\section{7. ¿Qué implicaciones tiene este estudio para la prevención de riesgos labora- les? ¿Cuál sería la recomendación para mejorar la práctica profesional en rela- ción con problema estudiado?}

De nuestro estudio se desprenden algunas ideas que creemos muy importantes, sobre todo, y como se está poniendo de manifiesto en otros ámbitos, el papel de la diversidad. No se accidentan más los más diferentes a nosotros. Nuestros resultados son claros, las diferencias culturales en términos de idioma y de distancia cultural no se asocian con un mayor número de accidentes.

En este sentido, los programas de intervención en lugar de intentar reducir las diferencias culturales entre la población nativa y los inmigrantes, deberían centrarse en identificar y neutralizar el peso de los valores culturales que sí se han mostrado relacionados con los accidentes. Dado que la población activa española también 
tiene accidentes, un programa de intervención enfocado en reducir las diferencias culturales entre locales e inmigrantes reduciría las tasas de accidentes de unos inmigrantes, pero aumentaría las de otros. Sin embargo, un programa de intervención enfocado en las dimensiones culturales que se han mostrado asociadas con accidentes sería mucho más efectivo. No se trata de hacer que los extranjeros se asemejan a los locales, sino de identificar, tanto en los extranjeros como en los locales, aquellas dimensiones culturales que están más relacionadas con la prevención y la seguridad en el trabajo.

\section{Resumen del artículo(1)}

Aunque las tasas de accidentes laborales en los inmigrantes suelen ser más altas que las de los trabajadores nativos, se ha realizado poca investigación sobre este tema desde una perspectiva cultural. El objetivo de este estudio es doble. Primero, explorar las diferencias culturales entre el país de acogida, España en este caso, y los países de origen de los inmigrantes, en cuanto al idioma y la distancia cultural, y analizar si estas diferencias están asociadas con sus tasas de accidentes laborales. En segundo lugar, esperamos probar los efectos lineales, cuadráticos y de interacción de los valores culturales, evaluados según el modelo de Hofstede (2001), sobre los accidentes laborales según el sector económico (agricultura, industria, construcción y servicios). Se analizaron las tasas de accidentes de trabajadores inmigrantes de 38 países en España. Se estimó la distancia cultural entre países (países de origen y de acogida - España) y se usó el análisis de regresión para probar asociaciones lineales, curvilíneas y de interacción. Los resultados muestran que hablar un idioma diferente y ser culturalmente diferentes no influye en los accidentes en los inmigrantes, excepto en la agricultura, donde hablar un idioma diferente se asocia con menos accidentes.

Los valores culturales nacionales (individualismo, distancia de poder, evitación de la incertidumbre, masculinidad y orientación a largo plazo) están relacionados con accidentes, ya sea linealmente (como la evitación de incertidumbre en la agricultura y la orientación a largo plazo en la industria, construcción y servicios) o cuadráticamente (como la distancia de poder y la evitación de la incertidumbre, en construcción y servicios), o se modulan entre sí (la distancia de poder modula el individualismo en la industria). Los valores culturales en sí mismos, en lugar del idioma o la distancia cultural entre países, son mejores predictores de la variación de los accidentes laborales en los inmigrantes. Se analizan las similitudes y diferencias entre los sectores económicos y se hacen propuestas para mejorar los programas de intervención para prevenir accidentes.

\section{Referencias}

1. García-Arroyo JA, Osca Segovia A. Occupational accidents in immigrant workers in Spain: The complex role of culture. Safety Science. 2020;121:507-515. doi:10.1016/j.ssci.2019.09.027 